\title{
Free vibration characteristics of cylindrical shells using a wave propagation method
}

\author{
A. Ghoshal ${ }^{a, *}$, S. Parthan ${ }^{\text {b }}$, D. Hughes ${ }^{\mathrm{c}}$ and \\ M. J. Schulz ${ }^{\mathrm{d}}$ \\ ${ }^{\mathrm{a}}$ Aerospace Research Engineer, Aerospace Structures \\ Group, NASA Center for Aerospace Research, North \\ Carolina A\&T State University, 1601 E. Market St., \\ Greensboro, NC 27410, USA \\ Tel: +1 336334 7255; Fax: +1 336334 7417; \\ E-mail: ghoshal@ncat.edu \\ ${ }^{\mathrm{b}}$ Department of Aerospace Engineering, Indian \\ Institute of Technology, Kharagpur, \\ Kharagpur-721302, India \\ Email: parthan@aero.iitkgp.ernet.in \\ ${ }^{\mathrm{c}}$ Graduate Research Assistant, McNair Research \\ Fellow, Structural Dynamics and Control Laboratory, \\ North Carolina A\&T State University, 1601 E. Market \\ St., Greensboro, NC 27410, USA \\ Tel.: + 13363347260 x606; Fax: +1 336334 7417; \\ Email:dhughes@ncat.edu \\ ${ }^{\mathrm{d}}$ Department of Mechanical Engineering, North \\ Carolina A\&T State University, 1601 E. Market St., \\ Greensboro, NC 27410, USA \\ Tel.: +1 3363347260 x313; Fax: +1 336334 7417; \\ E-mail: schulz@ncat.edu
}

In the present paper, concept of a periodic structure is used to study the characteristics of the natural frequencies of a complete unstiffened cylindrical shell. A segment of the shell between two consecutive nodal points is chosen to be a periodic structural element. The present effort is to modify Mead and Bardell's approach to study the free vibration characteristics of unstiffened cylindrical shell. The Love-Timoshenko formulation for the strain energy is used in conjunction with Hamilton's principle to compute the natural propagation constants for two shell geometries and different circumferential nodal patterns employing Floquet's principle. The natural frequencies were obtained using Sengupta's method and were compared with those obtained from classical ArnoldWarburton's method. The results from the wave propagation method were found to compare identically with the classical methods, since both the methods lead to the exact solution of the same problem. Thus consideration of the shell segment between two consecutive nodal points as a periodic structure is validated. The variations of the phase constants at the lower bounding frequency for the first propagation band for different nodal patterns have been computed. The method is highly computationally efficient.

\section{Introduction}

Circular cylindrical shells are commonly used as primary structural components in aerospace and naval structures. The problem of determining the vibration characteristics of cylindrical shells of finite length has been of interest to engineers and scientists for more than a century. Study of vibration characteristics of thin cylindrical shells, including the effects of stiffeners; have been done extensively by different authors using conventional methods $[7,8,13-15,19-25]$. Leissa [1] provides an excellent in depth review of the literature, theory and the models developed to study shell vibration. Wave propagation methods in periodically stiffened cylindrical shells with constant radii, has been studied by various authors $[3-7,11,17,20,21]$. Mead and Bardell [7,19-21] pioneered in this field, however comparison of their results with the existing literature is not reported [7]. Accorsi et al. [3,17,18] has applied wave propagation in conjunction with the FEM to analyze vibration characteristics of shells. A brief introduction in periodic systems and periodic structures is given in Appendix A.

\subsection{Present research work}

In this paper, the wave propagation method is used to study the vibration of cylindrical shells with a view to compare the results with well-known data. The intent of the paper has been to extend the concept of wave propagation in periodic structures to study free vibration characteristics in unstiffened structures and validate the results with existing classical shell vibration

\footnotetext{
*Corresponding author.
} 
results. The rationale of periodicity had been extended beyond conventional ideas of geometric and material periodicity to periodicity in free vibrational modes and nodal positions. The panel (segment of the shell) between a pair of consecutive circumferential nodes has been considered as a periodic element. The whole shell is analyzed as a one-dimensional periodic system with four degrees of freedom between each periodic element in the circumferential direction. The properties of each periodic element are related to its neighboring element via Floquet's principle involving propagation constants. The governing equations are set up using the strain and kinetic energy expressions. Propagation constants are computed for two different shell geometries for which results from other methods are available. The results from the wave propagation method are found to compare identically with the classical methods since both the methods lead to the exact solution of the same problem. Also a variation of the phase constants at the lower bounding frequency for the first propagation band for different nodal patterns has been observed.

\section{Model of shell vibration}

The whole structure will be regarded as an assembly of periodic elements joined end-to-end around the circumference of the cylindrical shell. The assumptions in the model are:

(i) Those wave motions are considered which propagate along and around the cylinder between one pair of circumferential nodes (Figs 1-2).

(ii) The nodes provide a simple support to the shell. This implies that the motion of the shell can be assumed to vary sinusoidally in space between the two consecutive circumferential nodes. In the case of an unstiffened shell the periodic element is defined to be the segment of the shell between two consecutive nodal positions.

The displacement along the junction line of adjacent periodic elements is completely defined by four co-ordinates $u, v, w$ and $\delta w / \delta \theta$, which are assumed to vary in $\mathrm{x}$-direction in proportion to $\cos (n \pi x / l)$ and $\sin (n \pi x / l)$ as appropriate. The four coupling coordinates between adjacent periodic elements are associated with coupling force or moment resultants $[P]$. These are respectively the longitudinal shear force $L$, tangential tension $Q$, radial shear force $S$ and the bending moment $M$. The displacement vector at each node is $\{q\}=\{u, v, w, \delta w / \delta \theta\}$ which is a $(1 \times 4)$ column vector of displacements (Fig. 1). The vector $\{P\}=\{L, Q, S, M\}$ is a $(1 \times 4)$ column vector of force and moment resultants.

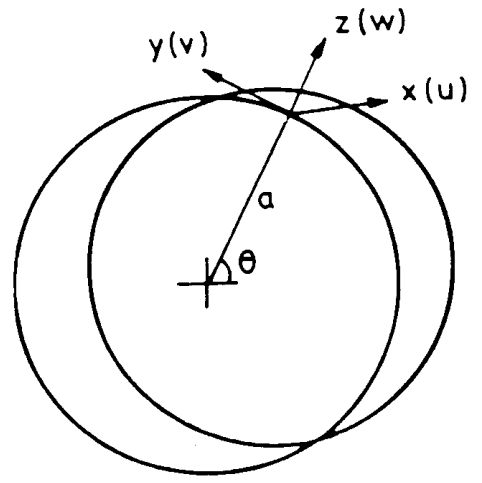

Fig. 1. Pictorial representation of the co-ordinate system used.
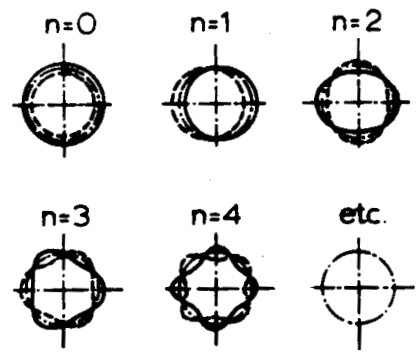

Fig. 2. Mode shape representation of cylindrical shell.

\subsection{Determination of propagation constants}

The analysis illustrates Mead and Bardell's formulation, which has been suitably modified to compute the propagation constants for two different unstiffened shell geometry. The formulation had been modified to remove the effects of the stiffeners and taking into account the new assumptions $\{$ (i) \& (ii) $\}$ as noted above. The structure has effectively been reduced to a onedimensional periodic system having four coupling coordinates between adjacent elements. Thus, four pairs of opposite going free harmonic waves can exist at any frequency. It has already been found that for a stiffened shell, two of these pairs are predominantly flexural wave, one is a predominantly shear wave and one is a predominantly longitudinal (in-plane) wave [7].

Each of these waves is associated with a particular propagation constant $\mu$. When one of the harmonic waves exists in the structure, the harmonic motion at one point in one of the periodic element is equal to exp $(\mu)$ times the motion of the corresponding point in the adjacent element [from Floquet's principle]. The propagation constant $\mu$ can be purely real, purely imaginary or generally complex $[2,16]$. 
The forces and moments at the corresponding points in adjacent periodic element are also related in the same way. In particular, the forces and moments at the left hand side of the adjacent periodic element are thus related. The whole set of edge displacements and force/moment resultants acting on the edges of the element are related by the state vector equation.

$$
\begin{aligned}
& {\left[M_{\Theta}, S_{\Theta}, Q_{\Theta}, L_{\Theta}, w_{1}, \Theta, w_{\Theta}, v_{\Theta}, u_{\Theta}\right]^{T}=} \\
& \exp (\mu)\left[-M_{0},-S_{0},-Q_{0},-L_{0}, w_{10}, w_{0}, v_{0}, u_{0}\right]^{T}
\end{aligned}
$$

or,

$$
\left[F_{\Theta}\right]=\exp (\mu)\left[F_{0}\right]
$$

where $\left[F_{\Theta}\right]$ and $\left[F_{0}\right]$ represent the state vectors, $M=$ Bending moment (Nm), $L=$ Longitudinal Shear Force $(\mathrm{N}), Q=$ Tangential Shear Force $(\mathrm{N}), S=$ Radial Shear Force $(\mathrm{N})$ and $\Theta=$ angle subtended by the periodic element. The individual elemental relationships in Eq. (1) are derived from Bloch's Theorem (Floquet's Principle) (see Appendix A).

Now the motion of the shell as a function of circumferential co-ordinate $\theta$, will be found to be governed by an eighth-order differential equation in $u, v$ or $w$. For a given value of $n$, this means these displacements can be expressed as sums of eight terms as follows:

$$
\begin{aligned}
& u=\sum_{m=1}^{8} A_{m n} \exp \left(\lambda_{m n} \theta\right) \cos (n \pi x / l) \exp (i \omega t) \\
& v=\sum_{m=1}^{8} B_{m n} \exp \left(\lambda_{m n} \theta\right) \sin (n \pi x / l) \exp (i \omega t) \\
& w=\sum_{m=1}^{8} C_{m n} \exp \left(\lambda_{m n} \theta\right) \cos (n \pi x / l) \exp (i \omega t)
\end{aligned}
$$

The eight $\lambda_{m n}$ 's occurred as four pairs of equal and opposite values and for a given frequency are found from the appropriate characteristic equation. The derivation of the shell equations of motion is based upon Love-Timoshenko's equation of motion for the cylindrical shell. The strain energy equation of a thin cylindrical shell according to the Love-Timoshenko's equation is as follows,

$$
\begin{aligned}
U= & \int_{0}^{t} \int_{0}^{l} \frac{E h}{2(1-v)^{2}}\left[\left(\frac{\partial u}{\partial x}\right)^{t}\right. \\
& +\frac{1}{a}\left(\frac{\partial v}{\partial \theta}+w\right)^{2}+\frac{2 v}{a} \frac{\partial u}{\partial x}\left(\frac{\partial v}{\partial \theta}+w\right)
\end{aligned}
$$

$$
\begin{aligned}
& +\frac{1}{2}(1-v)\left(\frac{\partial v}{\partial x}+\frac{1}{a}\left(\frac{\partial u}{\partial \theta}\right)\right)^{2} \\
& +\frac{h^{2}}{12}\left\{\left(\frac{\partial^{2} w}{\partial x^{2}}\right)^{2}+\frac{1}{a^{4}}\left(\frac{\partial^{2} w}{\partial \theta^{2}}-\frac{\partial v}{\partial \theta}\right)^{2}\right. \\
& +\frac{2 v}{a^{2}} \frac{\partial^{2} w}{\partial w^{2}}\left(\frac{\partial^{2} w}{\partial \theta^{2}}-\frac{\partial v}{\partial \theta}\right) \\
& \left.\left.+\frac{2(1-v)}{a^{2}}\left(\frac{\partial^{2} w}{\partial x \partial \theta}-\frac{\partial v}{\partial x}\right)^{2}\right\}\right] a \partial x / \partial \theta
\end{aligned}
$$

This kinetic energy expression is given by the standard expression:

$$
\begin{aligned}
T= & \frac{1}{2} \rho h \int_{0}^{t} \int_{0}^{l}\left\{\left(\frac{\partial u}{\partial t}\right)^{2}+\left(\frac{\partial v}{\partial t}\right)^{2}\right. \\
& \left.+\left(\frac{\partial w}{\partial t}\right)^{2}\right\} a \partial x \partial \theta
\end{aligned}
$$

in which rotational inertia terms are not included. Using Eqs (4) and (5) in conjunction with Hamilton's Principle the equations of motion for the shell are derived. Hamilton's Principle is

$$
\delta \int_{t_{1}}^{t_{2}}(T-U) d t=0
$$

This can be written in the matrix form as:

$$
\left[\begin{array}{lll}
D_{11} & D_{12} & D_{13} \\
D_{21} & D_{22} & D_{23} \\
D_{31} & D_{32} & D_{33}
\end{array}\right]\left\{\begin{array}{c}
u \\
v \\
w
\end{array}\right\}=\left\{\begin{array}{l}
0 \\
0 \\
0
\end{array}\right\}
$$

where

$$
\begin{aligned}
D_{11}= & E_{1}\left[2 D_{x x}+\frac{(1-v)}{a^{2}}\right] D_{\theta \theta}-\rho D_{t t}, \\
D_{12}= & E_{1}\left[\frac{1+v}{a}\right] D_{x t}, \quad D_{13}=E_{1} \frac{2 v}{a} D_{x} \\
D_{22}= & E_{1}\left[(1-v)(1-4 \beta) D_{x x}\right. \\
& \left.+\frac{2}{a^{2}}(1+\beta) D_{x x}\right]-\rho D_{t t} \\
D_{23}= & E_{1}\left[\frac{1}{a^{2}} D_{\theta}+2 \beta a^{2} D_{x x x}\right. \\
& \left.+4 \beta D_{x x \theta \theta}+\frac{2 \beta}{a^{2}} D_{\theta \theta \theta \theta}\right]+\rho D_{t t} \\
D_{33}= & {\left[\frac{2}{a^{2}}+2 \beta a^{2} D_{x x x x}+4 \beta D_{x x \theta \theta}\right.} \\
& \left.+\frac{2 \beta}{a^{2}} D_{\theta \theta \theta \theta}\right]+\rho D_{t t} \\
D_{21}= & D_{12}, \quad D_{31}=D_{13}, \quad D_{23}=D_{32}
\end{aligned}
$$




$$
\begin{aligned}
& E_{1}=\frac{E}{2(1-v)}, \quad \beta=\frac{h_{c}^{2}}{12 a^{2}}, \quad D_{x \theta}=\frac{\partial^{2}}{\partial x \partial \theta} \\
& D_{x}=\frac{\partial}{\partial x}, \quad D_{\theta}=\frac{\partial}{\partial \theta}, \quad D_{t t}=\frac{\partial^{2}}{\partial t^{2}} \ldots[7] .
\end{aligned}
$$

The $D$ 's are linear differential operators involving $\frac{\partial}{\partial x}, \frac{\partial}{\partial \theta}, \frac{\partial}{\partial t}$ etc., together with geometric mass and elastic properties of the shell. When the edges of the shell at $x=0$ and $x=l$ are simply supported, these equations are satisfied by,

$$
\begin{aligned}
u= & \sum_{m=1}^{\infty} \sum_{n=1}^{\infty} A_{m n} \exp \left(\lambda_{m n} \theta\right) \cos (n \pi x / l) \\
& \exp (i \omega t) \\
v= & \sum_{m=1}^{\infty} \sum_{n=1}^{\infty} B_{m n} \exp \left(\lambda_{m n} \theta\right) \sin (n \pi x / l) \\
& \exp (i \omega t) \\
w= & \sum_{m=1}^{\infty} \sum_{n=1}^{\infty} C_{m n} \exp \left(\lambda_{m n} \theta\right) \cos (n \pi x / l) \\
& \exp (i \omega t)
\end{aligned}
$$

Substitution of just one of the set of $(8 \times \infty)$ terms in the double series of Eq. (9) transforms the differential operators into functions of $x_{m n}, n$ and $\omega$. These functions will be denoted by $b_{11}, b_{12}, b_{13}^{\prime} \ldots b_{33}$. So Eq. (7) becomes,

$$
\left[\begin{array}{lll}
b_{11} & b_{12} & b_{13} \\
b_{21} & b_{22} & b_{23} \\
b_{31} & b_{32} & b_{33}
\end{array}\right]\left\{\begin{array}{c}
u \\
v \\
w
\end{array}\right\}=\left\{\begin{array}{l}
0 \\
0 \\
0
\end{array}\right\}
$$

where,

$$
\begin{aligned}
& b_{11}=a_{1}+b \lambda_{m n}, \quad b_{21}=b_{12}, \quad b_{31}=b_{13} \\
& b_{12}=c \lambda_{m n}, \quad b_{22}=f+e \lambda_{m n}^{2}, \\
& b_{32}=g \lambda_{m n}^{2}+h_{1} \lambda_{m n} \\
& b_{13}=d, \quad b_{23}=b_{32} \\
& b_{33}=j+k \lambda_{m n}^{2}+l_{1} \lambda_{m n}^{4} \\
& a_{1}=E_{1} 2\left(\frac{n \pi}{l}\right)^{2}-\rho \omega^{2}, \\
& b=-\left[\frac{1-v}{a^{2}}\right] E_{1}, \quad c=-E_{1}\left(\frac{1+v}{a}\right) \frac{n \pi}{l} \\
& d=E_{1}\left(\frac{2 v}{a}\right) \frac{n \pi}{l}, \quad e=E_{1}\left(\frac{2 v}{a^{2}}\right)(1+\beta), \\
& f=-E_{1}(1-v)(1+4 \beta)\left(\frac{n \pi}{l}\right)+\rho \omega^{2}
\end{aligned}
$$

$$
\begin{aligned}
& g=E_{1}\left\{\left(\frac{2}{a^{2}}\right)+2 \beta(2-v)\right\}\left(\frac{n \pi}{l}\right)^{2}, \\
& h=-2 \beta \frac{E_{1}}{a^{2}} \\
& j=E_{1}\left\{\left(\frac{2}{a^{2}}\right)+2 \beta a^{2}\left(\frac{n \pi}{l}\right)^{2}\right\}-\rho \omega^{2} \\
& k=-E_{1} 4 \beta\left(\frac{n \pi}{l}\right)^{2}
\end{aligned}
$$

Upon expanding the determinant we have,

$$
\begin{aligned}
& A\left(\lambda_{m n}^{2}\right)^{4}+B\left(\lambda_{m n}^{2}\right)^{3}+C\left(\lambda_{m n}^{2}\right)^{2} \\
& +D\left(\lambda_{m n}^{2}\right)+E_{3}=0
\end{aligned}
$$

wherein terms of the characters defined above are:

$$
\begin{aligned}
A= & b e l_{1}-b h_{1}^{2} \\
B= & a_{1} e l_{1}-a_{1} h^{2}+b f l_{1}-b e k-2 b g h_{1}-c^{2} l_{1} \\
C= & a_{1} f l_{1}+a_{1} e k-2 a_{1} g h_{1}+b f k+b e j \\
& -b g^{2}-c^{2} k+2 c d h_{1} \\
D= & a_{1} f k+a_{1} e j-a_{1} g^{2}+b f j-c^{2} j \\
& +2 c d g-d^{2} e \\
E_{3}= & a_{1} f j-d^{2} f
\end{aligned}
$$

$\lambda=$ circumferential wave number $\left(\mathrm{m}^{-1}\right), v=$ Poisson's ratio, $E=$ Young's modulus of elasticity $\left(\mathrm{Nm}^{-2}\right)$, $l=$ length of shell, $a=$ mean shell radius, $h=$ shell thickness, $t=$ time variable (sec), and $\rho=$ material density $\left(\mathrm{kg} / \mathrm{m}^{3}\right)$. The solution of the bi-quartic Eq. (12) yields four (in general complex) equal and opposite values of $\lambda_{m n}$. The roots for the solution of vibration problem will usually have the form $\lambda_{m n}= \pm a, \pm i b, \pm(c \pm i d)$ where $a, b, c$ and $d$ are real quantities.

For a finite shell, there will always be at least 2 roots of the form $( \pm i b)$. For non-trivial solutions for $A_{m n}, B_{m n}$, and $C_{m n}$, the determinant of the matrix of Eq. (9) must vanish. When the determinant is expanded, an eighth-order polynomial equation is found for $\lambda_{m n}$ involving $n$ and $\omega^{2}$ in the coefficients. When any one of the eight roots is known, it may be substituted back into Eq. (9) to find $A_{m n}, B_{m n}$, in terms of $C_{m n}$. Thus

$$
\begin{aligned}
A_{m n} / C_{m n}= & \left(b_{12} b_{33}-b_{13} b_{23}\right) / \\
& \left(b_{11} b_{33}-b_{13} b_{12}\right)=\phi_{m n} \\
B_{m n} / C_{m n}= & \left(b_{13}^{2}-b_{11} b_{33}\right) / \\
& \left(b_{11} b_{33}-b_{13} b_{12}\right)=\Psi_{m n}
\end{aligned}
$$


These terms characterize the particular type of wave motion, giving the ratio of the longitudinal or circumferential displacement to the flexural displacement. This unique relationship amongst $A_{m n}, B_{m n}$, and $C_{m n}$ had been developed. This means that the each displacement, force and moment in the two state vectors can be expressed in terms of just one of the sets of coefficients for $A_{m n}, B_{m n}$, and $C_{m n}$. The two state vectors may very well be expressed as:

$$
\begin{aligned}
\left\{F_{\Theta}\right\} & =\left[K_{\Theta}\right]\left[C_{m n}\right]_{\cos }^{\sin }(n \pi x / l) e^{j \omega t} \\
\left\{F_{0}\right\} & =\left[K_{0}\right]\left[C_{m n}\right]_{\cos }^{\sin }(n \pi x / l) e^{j \omega t}
\end{aligned}
$$

in which $\sin (n \pi x / l)$ is used in conjunction with $M_{0, \Theta}, S_{0, \Theta}, Q_{0, \Theta}, v_{0, \Theta}, w_{0, \Theta}, w_{10, \Theta}$ and $\cos (n \pi x / l)$ is used with $L_{0, \Theta}, u_{0, \Theta} \cdot\left[k_{0}\right]$ and $\left[K_{\Theta}\right]$ complex square stiffness matrices. Putting Eq. (12) into Eq. (1) yields:

$$
\left[K_{\Theta}\right]\left[C_{m n}\right]=e^{\mu}\left[K_{\Theta}\right]\left[C_{m n}\right]
$$

or,

$$
\left[K_{0}\right]^{-1}\left[K_{\Theta}\right]\left[C_{m n}\right]=e^{\mu}[I]\left[C_{m n}\right]
$$

This equation is in canonical form for the determination of the eigenvalues $\exp (\mu)$ of the matrix product $\left[K_{0}\right]^{-1}\left[K_{\Theta}\right]$. The complex propagation constantsare then found from these eigenvalues $[7,8]$.

\subsection{Resultants on the edges of the periodic element}

The stress and moment resultants are the forces and moments per unit length along the edge corresponding to the displacement field $u, v$, and $w$. They include contributions from Hamilton's Principle, which states,

$$
\delta \int_{t_{1}}^{t_{2}}(T-U+W) d t=0
$$

Here $T$ and $U$ are the kinetic and potential energy of all the components of the periodic element and $W$ is the work done by the force-moment resultants in Joules. The expression for the potential energy of the cylindrical shell corresponding to the Love-Timoshenko theory is given in Eq. (4). Substituting the energy expressions into Eq. (14) leads to the three equations of motions of the shell and eight boundary conditions for the two edges, four for each edge. These boundary conditions relate the local force or moment resultants applied at any point $\mathrm{x}$-along the edge to the shell properties and to their displacements and displacement derivatives at the edges. When one of these equations is used to give the resultant at $\theta=0$ or $\theta=\Theta$, the sign of shell differs in two cases. The modified eight boundary conditions for the edges of the shell segment are expressed by the following four equations,

$$
\begin{aligned}
M_{0, \Theta}= & \left.E_{4}\left[\frac{1}{a^{2}}\left(w_{\theta \theta}-v_{\theta}\right)+v\left(w_{x x}\right)\right]\right|_{0} ^{\Theta} \\
S_{0, \Theta}= & -E_{4}\left[\frac{1}{a^{3}}\left(w_{\theta \theta \theta}-v_{\theta \theta}\right)-2 \frac{(1-v)}{a} v_{\theta \theta}\right. \\
& \left.+\frac{2-v}{a} w_{x x \theta}\right]\left.\right|_{0} ^{\Theta} \\
Q_{0, \Theta}= & E_{5}\left[\frac{2}{a}\left(v_{\theta}+w\right)+2 v u_{x}-\frac{h}{12^{2}}\right. \\
& \left.\left\{\frac{2}{a^{3}}\left(w_{\theta \theta}-v_{\theta}\right)+\frac{2 v}{a} w_{\theta \theta}\right\}\right]\left.\right|_{0} ^{\Theta} \\
L_{0, \Theta}= & \left.E_{5}\left[(1-v)\left(v_{x}+\frac{1}{a} \mu \theta\right)\right]\right|_{0} ^{\Theta}
\end{aligned}
$$

where

$$
\begin{aligned}
& E_{4}=\frac{E h^{2}}{12\left(1-v^{2}\right)}, \quad E_{5}=\frac{E h}{2\left(1-v^{2}\right)}, \\
& w_{\theta \theta}=\frac{\partial^{2} w}{\partial \theta^{2}}, \quad v_{\theta}=\frac{\partial v}{\partial \theta} \ldots[7]
\end{aligned}
$$

When $u, v, w$ are given by Eq. (3), the above force resultants are expressed in terms of $A_{m n}, B_{m n}$, and $C_{m n}$. The two sets of 4 force/moment resultants are denoted by the vector $\left\{P_{0, \Theta}\right\}$ where

$$
\left\{P_{0, \Theta}\right\}^{\Theta}=\left\{M_{0, \Theta}, S_{0, \Theta}, Q_{0, \Theta}, L_{0, \Theta}\right\}
$$

Then from Eq. (4) these resultants are linearly related to the $A_{m n}$ 's, $B_{m n}$ 's, and $C_{m n}$ 's through,

$$
\begin{aligned}
\left\{P_{0, \Theta}\right\}= & {\left[\alpha_{0, \Theta}\right]\left[A_{m n}\right]+\left[\zeta_{0, \Theta}\right]\left[B_{m n}\right] } \\
& +\left[\xi_{0, \Theta}\right]\left[C_{m n}\right]
\end{aligned}
$$

The matrices $\alpha, \zeta$ and $\xi$ consist of algebraic terms derived from the derivative functions of $u, v$, and $w$ respectively in Eqs (15). Now

$$
\begin{aligned}
& {\left[A_{m n}\right]=\left[\phi_{m n}\right]\left[C_{m n}\right]} \\
& {\left[B_{m n}\right]=\left[\Psi_{m n}\right]\left[C_{m n}\right]}
\end{aligned}
$$

where the $\phi$ and $\Psi$ matrices are diagonal, with elements given by $\phi$ 's and $\Psi$ 's of Eq. (11). Therefore,

$$
\begin{aligned}
\left\{P_{0, \Theta}\right\}= & \left(\left[\alpha_{0, \Theta}\right]\left[\phi_{m n}\right]+\left[\zeta_{0, \Theta}\right]\left[\Psi_{m n}\right]\right. \\
& \left.+\left[\xi_{0, \Theta}\right]\right)\left[C_{m n}\right]
\end{aligned}
$$

Separately we have, 


$$
\begin{aligned}
{\left[P_{0}\right] } & =\left[K_{0 \Theta_{1}}\right]\left[C_{m n}\right] \\
{\left[P_{\Theta}\right] } & =\left[K_{\Theta \Theta_{1}}\right]\left[C_{m n}\right]
\end{aligned}
$$

where $\left[K_{0 \Theta_{1}}\right]$ and $\left[K_{\Theta \Theta_{1}}\right]$ are rectangular $(4 \times 8)$ matrices and form the top of the halves of $\left[K_{0}\right]$ and $K_{\Theta}$ $(8 \times 8)$ matrices of Eq. (13)

$$
\left[K_{0 \Theta_{1}}\right]=\{[D]+[E]+[F]\}
$$

and where

$$
\begin{aligned}
& {[D]=\left[\alpha_{0, \Theta_{1}}\right]\left[\phi_{m n}\right], \quad[E]=\left[\zeta_{0, \Theta_{1}}\right]\left[\Psi_{m n}\right],} \\
& {[F]=\left[\xi_{0, \Theta_{1}}\right]} \\
& \sum_{\substack{i=1 \\
j=1}}^{8}\left[D_{i j}\right]=\sum_{\substack{i=1 \\
j=1}}^{8}\left[\alpha_{i j}\right]\left[\phi_{i n}\right]
\end{aligned}
$$

For the unstiffened shell:

$$
\begin{aligned}
& j=1, \ldots, 8 . \\
& \alpha_{1 j}=0, \quad \alpha_{2 j}=0, \quad \alpha_{3 j}=-E_{5} 2 v \frac{n \pi}{l}, \\
& \alpha_{4 j}=-E_{5} \frac{\lambda_{m n}}{a}(1-v) \\
& \sum_{\substack{i=1 \\
j=1}}^{8}\left[E_{i j}\right]=\sum_{i=1}^{8}\left[\zeta_{i j}\right]\left[\Psi_{i n}\right] \\
& \zeta_{1 j}=-E_{4} \frac{\lambda_{m n}}{a^{2}}, \\
& \zeta_{2 j}=-E_{4}\left[\frac{-\lambda_{m n}^{2}}{a^{3}}+\frac{2(1-v)}{a}(n \pi / l)^{2}\right] \\
& \zeta_{3 j}=E_{5}\left(\frac{2 \lambda_{m n}}{a}+\frac{2 \beta \lambda_{m n}}{a}\right) \\
& \zeta_{4 j}=E_{5}(1-v) \frac{n \pi}{l} \\
& \sum_{\substack{i=1 \\
j=1}}\left[F_{i j}\right]=\sum_{i=1}^{8}\left[\xi_{i j}\right] \\
& \xi_{1 j}=E_{4}\left[\frac{\lambda_{m n}^{2}}{a^{2}}-v\left(\frac{n \pi}{l}\right)^{2}\right] \\
& \xi_{2 j}=-E_{4}\left[\frac{\lambda_{m n}^{3}}{a^{3}}-\frac{2(1-v)}{a}\left(\frac{n \pi}{l}\right)^{2}\right] \\
& \xi_{3 j}=E_{5}\left[\frac{2}{a}-\alpha^{2} \beta\left(\frac{2 \lambda_{m n}^{2}}{a^{3}}+\frac{2 v}{a}\left(\frac{n \pi}{l}\right)^{2}\right)\right] \\
& \xi_{4 j}=0
\end{aligned}
$$

Similarly,

$$
\left[K_{\theta \Theta_{1}}\right]=\{[X]+[Y]+[Z]\}
$$

where

$$
[X]=\left[\alpha_{\Theta, \Theta_{1}}\right]\left[\phi_{m n}\right], \quad[Y]=\left[\zeta_{\Theta, \Theta_{1}}\right]\left[\Psi_{m n}\right],
$$

$$
[Z]=\left[\xi_{\Theta, \Theta_{1}}\right]
$$$$
\sum_{\substack{i=1 \\ j=1}}^{8}\left[X_{i j}\right]=\sum_{\substack{i=1 \\ j=1}}^{8}\left[\hat{\alpha}_{i j}\right]\left[\phi_{j n}\right] e^{\lambda_{j n} \theta}
$$$$
\hat{\alpha}_{1 j}=0, \quad \hat{\alpha}_{2 j}=0
$$$$
\hat{\alpha}_{3 j}=-E_{5} 2 v \frac{n \pi}{l} \text {, }
$$$$
\hat{\alpha}_{4 j}=-E_{5} \frac{\lambda_{m n}}{a}(1-v)
$$$$
\sum_{\substack{i=1 \\ j=1}}^{8}\left[Y_{i j}\right]=\sum_{\substack{i=1 \\ j=1}}^{8}\left[\hat{\zeta}_{i j}\right]\left[\Psi_{j n}\right] e^{\lambda_{j n} \theta}
$$

$\hat{\zeta}_{1 j}=-E_{4} \frac{\lambda_{m n}}{a^{2}}$,

$\hat{\zeta}_{2 j}=-E_{4}\left[\frac{-\lambda_{m n}^{2}}{a^{3}}+\frac{2(1-v)}{a}(n \pi / l)^{2}\right]$,

$\hat{\zeta}_{3 j}=E_{5}\left(\frac{2 \lambda_{m n}}{a}+\frac{2 \beta \lambda_{m n}}{a}\right)$

$\hat{\zeta}_{4 j}=E_{5}(1-v) \frac{n \pi}{l}$

$\sum_{\substack{i=1 \\ j=1}}^{8}\left[Z_{i j}\right]=\sum_{\substack{i=1 \\ j=1}}^{8}\left[\hat{\xi}_{i j}\right] e^{\lambda_{j n} \theta}$

$\hat{\xi}_{1 j}=E_{4}\left[\frac{\lambda_{m n}^{2}}{a^{2}}-v\left(\frac{n \pi}{l}\right)^{2}\right]$,

$\hat{\xi}_{2 j}=-E_{4}\left[\frac{\lambda_{m n}^{3}}{a^{3}}-\frac{2(1-v)}{a}\left(\frac{n \pi}{l}\right)^{2}\right]$,

$\hat{\xi}_{3 j}=E_{5}\left[\frac{2}{a}-\alpha^{2} \beta\left(\frac{2 \lambda_{m n}^{2}}{a^{3}}+\frac{2 v}{a}\left(\frac{n \pi}{l}\right)^{2}\right)\right]$,

$\hat{\xi}_{4 j}=0$

The lower parts of and are those transformation matrices which relate $\left[w_{0}, w_{1,0}, v_{0}, u_{0}\right]=\left\{q_{0}\right\}$ and $\left[w_{\Theta}, w_{1, \Theta}, v_{\Theta}, u_{\Theta}\right]=\left\{q_{\Theta}\right\}$ to $\left[C_{m n}\right]$. 

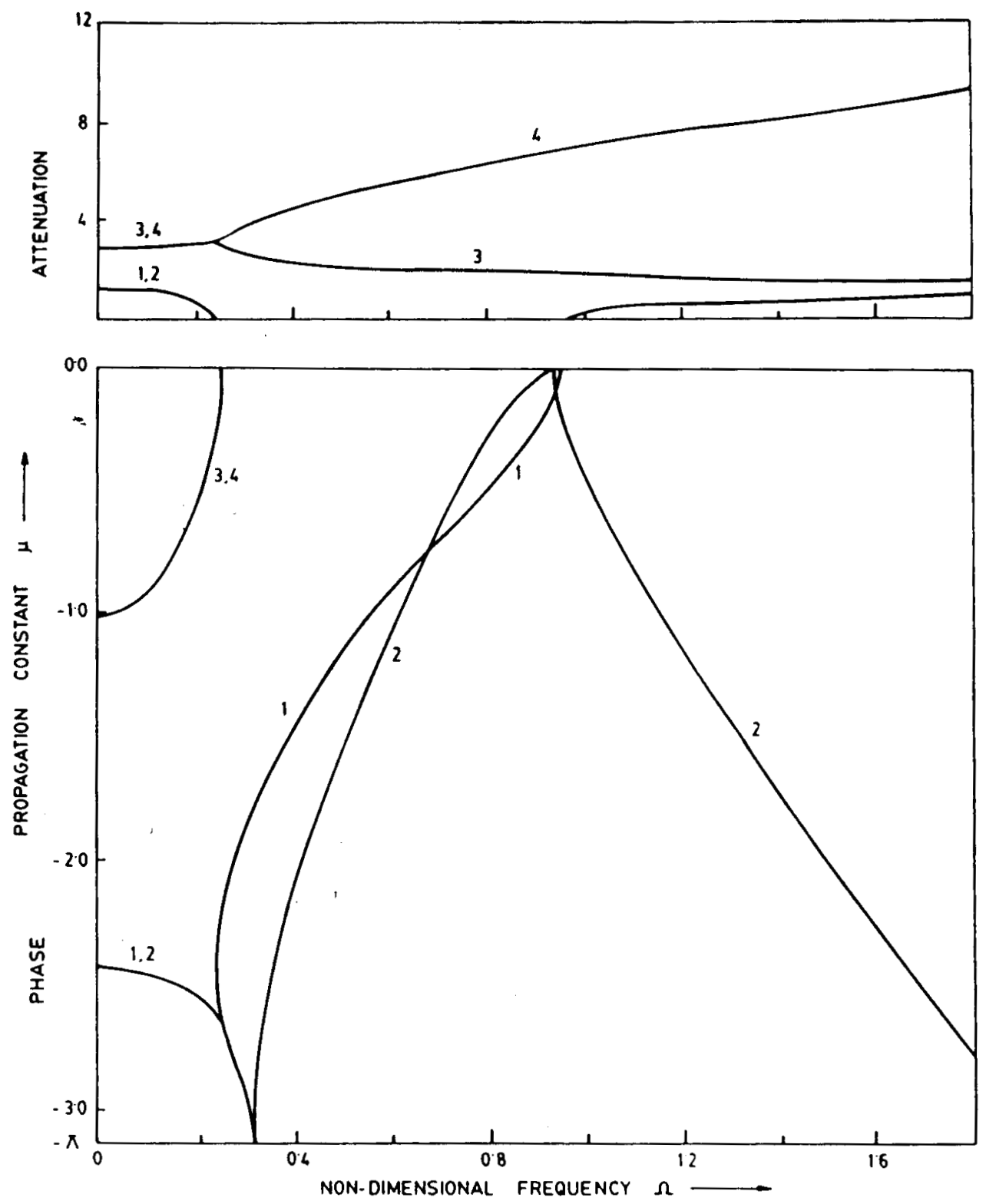

Fig. 3. Propagation constants for the cylindrical shell (Case 1).

\section{Evidently,}

$$
\left[q_{0}\right]=\left[K_{0 L}\right]\left[C_{m n}\right]
$$

where $\left[K_{0 L}\right]$ is a $(4 \times 8)$ rectangular matrix.

$$
\begin{aligned}
& K_{0 L_{1 j}}=1, \quad K_{0 L_{2 j}}=\lambda_{m n}, \\
& K_{0 L_{3 j}}=\Psi_{m n}, \quad K_{0 L_{4 j}}=\phi_{m n}, \\
& {\left[q_{\Theta}\right]=\left[K_{\Theta L}\right]\left[C_{m n}\right]}
\end{aligned}
$$

where $\left[K_{\Theta L}\right]$ is a $(4 \times 8)$ rectangular matrix.

$$
\begin{aligned}
& K_{\Theta L_{1 j}}=e^{\lambda_{m n} \theta}, \quad K_{\Theta L_{2 j}}=\lambda_{m n} e^{\lambda_{m n} \theta}, \\
& K_{\Theta L_{3 j}}=\Psi m n e^{\lambda_{m n} \theta}, \\
& K_{\Theta L_{4 j}}=\phi m n e^{\lambda_{m n} \theta}
\end{aligned}
$$

where $i=1, \ldots 4, j=1, \ldots 8, m=1, \ldots 8$.

The complete matrices $\left[K_{0}\right]$ and $\left[K_{\Theta}\right]$ are given by

$$
\left[K_{0}\right]=\left[\frac{K_{0 \Theta}}{K_{\Theta L}}\right]
$$




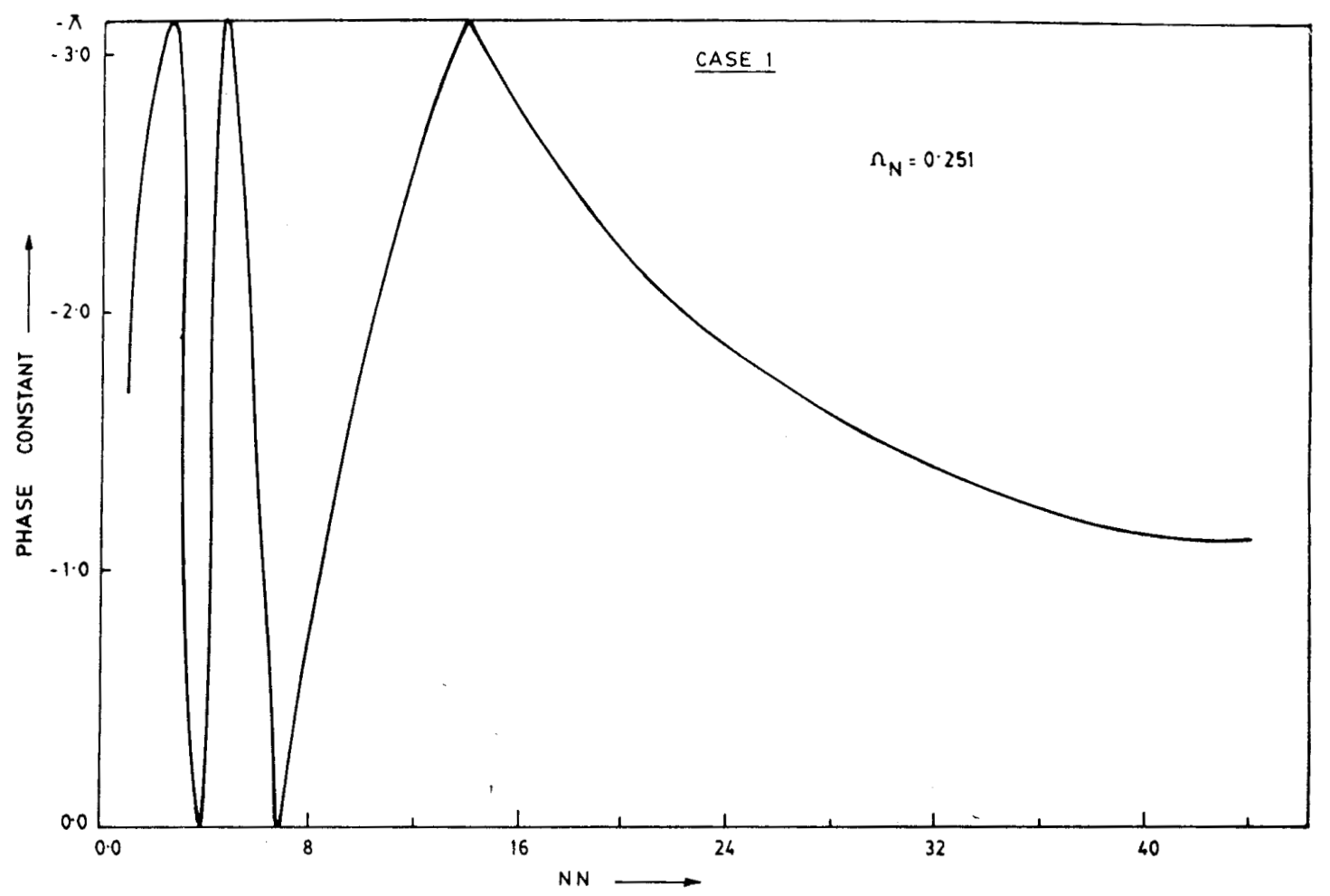

Fig. 4. Values of phase constant for different nodal patterns at the lowest bounding frequency (Case 1).

$$
\left[K_{\Theta}\right]=\left[\frac{K_{\Theta \Theta_{1}}}{K_{\Theta_{1} L}}\right]
$$

$\left[K_{0}\right]$ and $\left[K_{\Theta}\right]$ are $8 \times 8$ structural property matrices of the periodic element at $\theta=0$ and $\theta=\Theta$. These are now used in Eq. (13) to find the eigenvalues, $e^{\mu}$ and the propagation constants $\mu$.

\section{Discussion of results}

A non-dimensional frequency $\Omega$ has been used in Figs 3-8 to show how the propagation constants vary with frequency and other parameters: $\Omega_{N}=$ $\left[\rho a^{2} \omega^{2}\left(1-v^{2}\right) / E\right]^{1 / 2}$ where $\omega$ is the actual radian frequency $\left(s^{-1}\right)$. The other terms have been defined previously. Two separate computer programs were developed in F77 to generate the results for the present wave propagation method and the Arnold-Warburton's method.

\subsection{The propagation constant curves}

The patterns of propagation constant curves obtained from the unstiffened analysis [4] show a remarkable similarity in their trend to those obtained by Mead and Bardell [7] from the axially stiffened shell. At any frequency four distinctly different values occur. Over the lower frequency region curve marked 1,2 and 3,4 represents a complex conjugate pair. One curve in the real and one in the imaginary domain are shown. At higher frequencies, however, curves 1,3 do not represent a complex conjugate pair as in the case of the stiffened shell analysis. Each curve (or a pair of curves) has its negative counterpart meaning that eight propagation constants exist altogether at any frequency. Two cases of different shell geometries are studied.

\subsubsection{Case 1}

For the shell geometry chosen by Mead and Bardell [7] the shell properties are as follows: Length of the shell, $l=0.135 \mathrm{~m}$, radius of the shell, $a=0.381 \mathrm{~m}$, thickness of the shell, $h=0.000559 \mathrm{~m}$, number of halfwaves in axial direction along the shell length, $n=1$, damping factor $=0.0$. Figure 3 is representative of the several mode shapes studied. In this case the curves 3, 4 are complex conjugate pairs up to $\Omega_{N} \leqslant 0.251$ (Fig. 3). Both the curves are completely attenuating for $\Omega_{N}>0.251$. In the case of curve 4 , it 

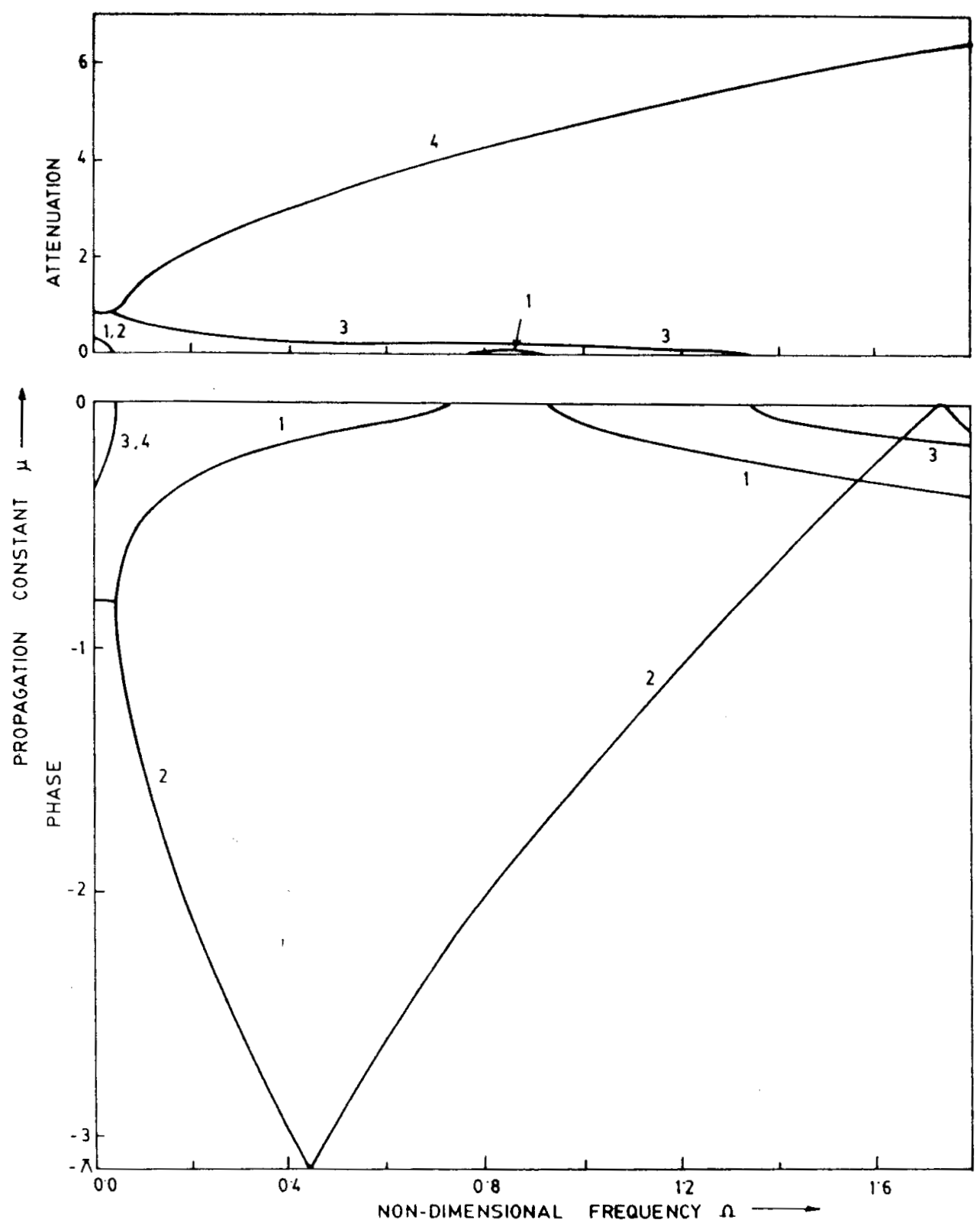

Fig. 5. Propagation constants for the cylindrical shell (Case 2).

is increasing in attenuation while decreasing steadily in the case of curve 3 . Both the curves 3 and 4 are primarily associated with the attenuating wave motion from $\Omega_{N}>0.251$ in which the shell displacements are predominantly radially flexural. Curves 1,2 are complex conjugate pairs up to $\Omega_{N}=0.251$ and after that both of them become propagating waves from $\Omega=0.251$ to $\Omega_{N}=0.955$. Curve 1 becomes an attenuating wave from $\Omega>0.955$ onwards while curve 2 continues to propagate at higher frequencies. From $\Omega_{N}=0.251$ to $\Omega_{N}=0.955$ the curve represents a complete propagation band. Curves 1,2 are predominantly associated with longitudinal and tangential wave motions. For lower circumferential modes it is being observed that curve 1,2 constantly varies its phase constant values from 0 and $\pi$ from $\Omega_{N}=0.251$ to $\Omega_{N}=0.955$. From $\Omega_{N} \geqslant 0.955$, however, only curve 2 is purely a propagating wave. Hence, here the first propagation band 


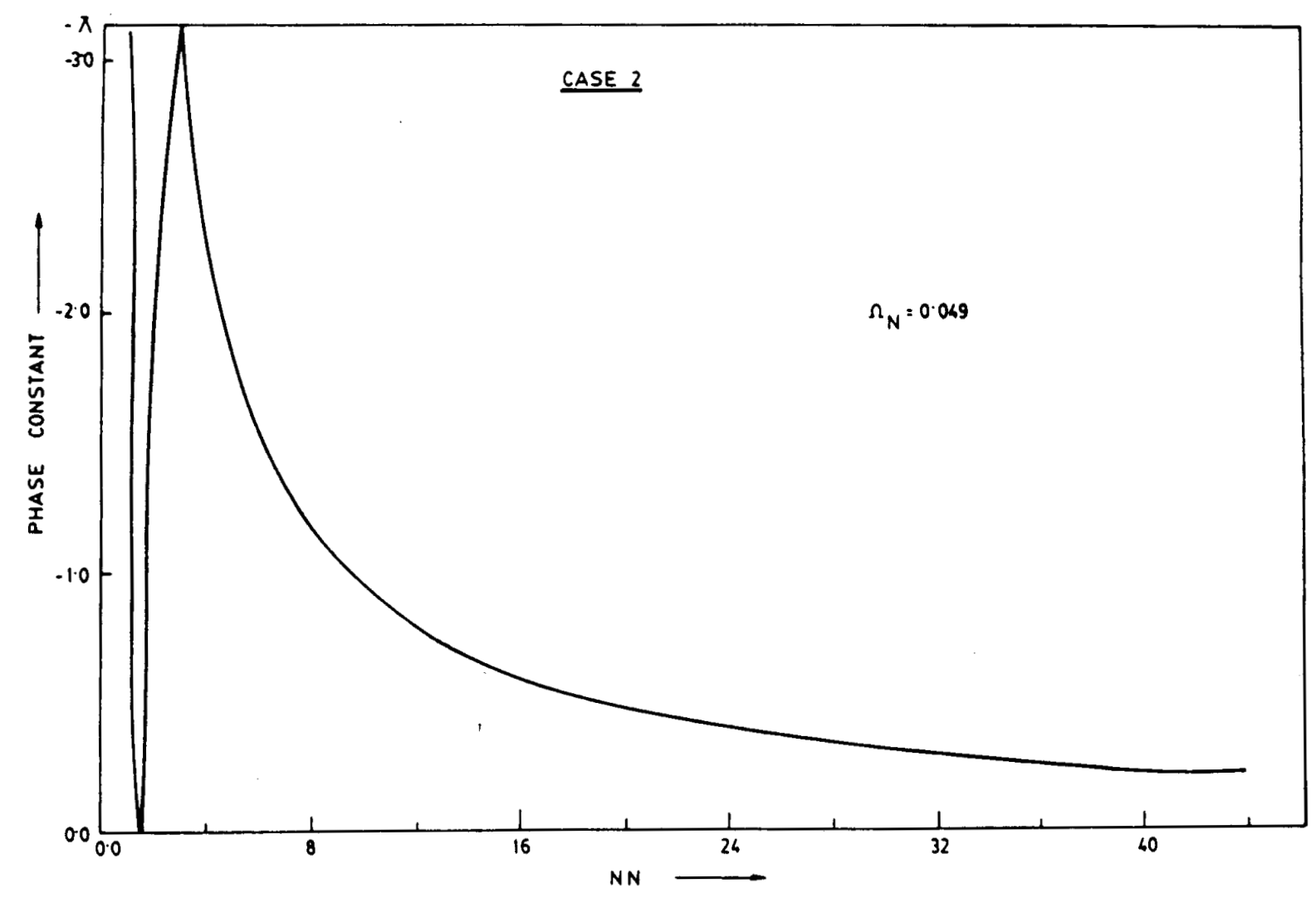

Fig. 6. Values of phase constant for different nodal patterns at the lowest bounding frequency (Case 2).

between $\Omega_{N}=0.251$ to $\Omega_{N}=0.955$ is not distinct. For higher mode shapes ( $m \geqslant 6$ ), however, the propagation band is highly distinct. However, unlike Mead and Bardell's [7] observation for the stiffened shell with axial stiffeners, here, it is observed that even for higher modal patterns, i.e., even when the size of the periodic element decreases, the lower and the upper bounding frequencies are fixed at $\Omega_{N}=0.251$ and $\Omega_{N}=0.955$ respectively. It is also observed that the phase constant for the lower frequency $\left(\Omega_{N}=0.251\right)$ of the propagation band varies between 0 and $\pi$ for different mode shapes while it is zero for the upper bounding frequencies $\left(\Omega_{N}=0.955\right)$. The phase constants at the lower bounding frequencies for the first propagation band at $\Omega_{N}=0.251$ are plotted against nodal patterns (Fig. 4). The curve touches $\pi$ between $N N=14$ and $N N=15$. After this the curve seems to follow a definite pattern. The product of the phase constant $\mu_{i}$ at lower bounding frequency $\left(\Omega_{N}=0.251\right)$ and the corresponding nodal pattern $N N$ is constant (here it is equal to 44.26$)$ for each mode shape $(N N \geqslant 15)$.

\subsubsection{Case 2}

The program for the unstiffened shell is then applied to another shell geometry [24]. For the shell geometry chosen by Parthan and Johns [24] the shell properties are as follows: Length of the shell, $l=0.60325 \mathrm{~m}$, radius of the shell, $a=0.24257 \mathrm{~m}$, thickness of the shell, $h=0.0007112 \mathrm{~m}$, number of halfwaves in axial direction along the shell length, $n=1$, damping factor $=0.0$. Figure 5 is representative of the several mode shapes studied. The observations (Fig. 5) are similar to the Case 1. The lower and the upper bounding frequencies for the first propagation band are at $\Omega_{N}=0.049$ and at $\Omega_{N}=0.75$. The curves follow a similar pattern as in Case 1. The curve drawn for the phase constants at the lower bounding frequency $\left(\Omega_{N}=0.049\right)$ against the nodal patterns $\mathrm{N}$ touches between $N N=3$ and $N N=4$. After this the curves seem to follow a definite pattern (Fig. 6). The product of the phase constant at lower bounding frequency $\left(\Omega_{N}=0.049\right)$ and the corresponding nodal pattern $N N$ is constant (here it is equal to 9.525$)$ for each mode shape $(N N \geqslant 4)$. 


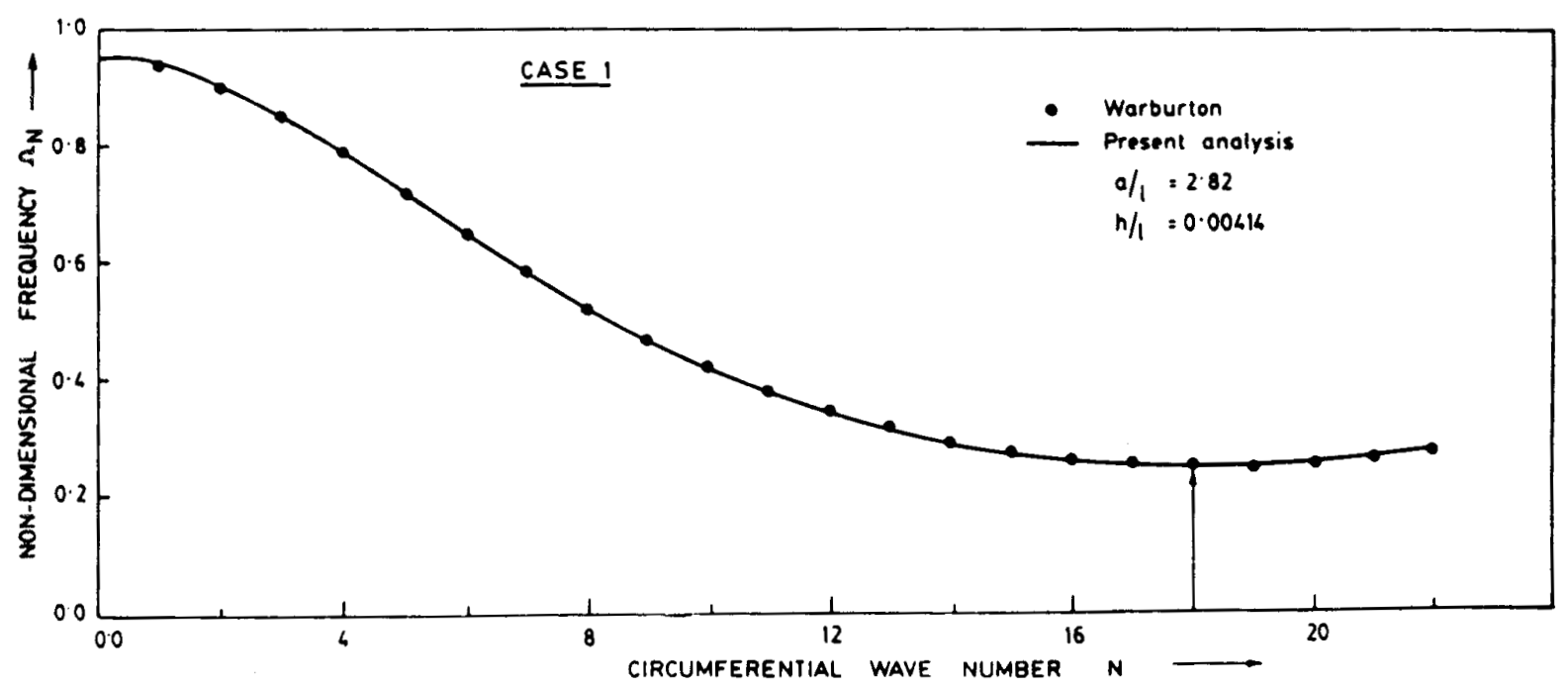

Fig. 7. Comparison of natural frequencies obtained by present analysis with those obtained from Warburton's method (Case 1).

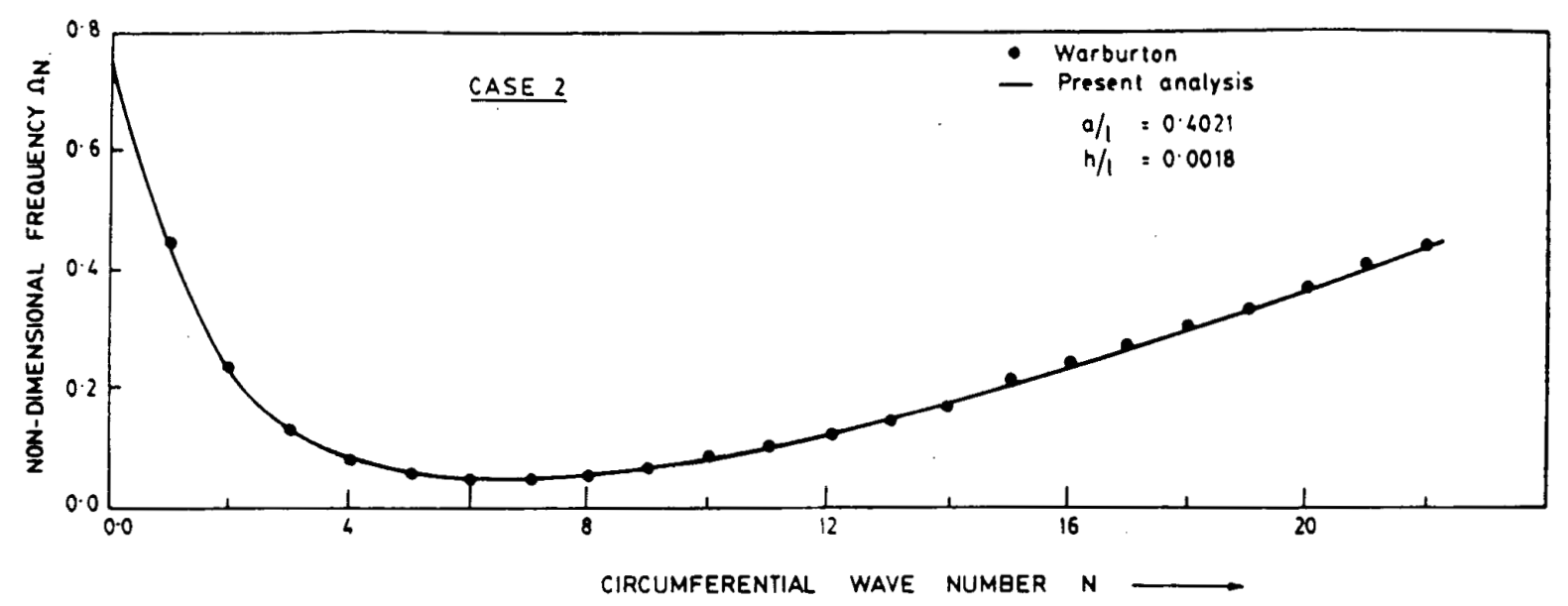

Fig. 8. Comparison of natural frequencies obtained by present analysis with those obtained from Warburton's method (Case 2).

\subsection{Natural frequencies of the closed shell}

The circumferential length of the periodic element for the Case 1 is $\theta=2 \pi(0.381) / 17=0.140 \mathrm{~m}$. In the Case $2, \theta=2(0.24257) / 11=0.139 \mathrm{~m}$. Now $2 \pi / \theta$ is equal to 44.6 and 45.2 for both the cases respectively. It should be noted here that we have considered comparative cases of Arnold-Warburton's method [1, $13,22]$ where the number of bays considered is 44 . The condition taken in the Arnold-Warburton's method is that of 'No Stiffening' to simulate the unstiffened condition. Arnold and Warburton [22] solved the complete boundary-value problem of free vibration of a finite cylindrical shell using strain energy calculations. The mode shapes for the above two cases considered are 17 and 11 respectively where Sengupta's method [12] is applied to compute the natural frequencies.

The natural frequencies of the unstiffened closed shell had been obtained using Sengupta's method [12]. A continuous cylindrical shell has an infinite number of natural frequencies. If, at a particular frequency, a propagating wave travels once around the whole shell and so undergoes a total phase change of $2 j \pi$ ( $j$ an integer), then that frequency is the natural frequency of free vibration of the closed shell. The natural mode is the superposition of two but equal opposite-going 
waves. If the propagation constant is $\mu_{i}$ (phase change per periodic distance) and there are $\mathrm{N}$ equal segments of the shell, then the condition for a natural mode to exist is $N_{\mu_{i}}=2 j \pi(j=0,1,2 \ldots N)$ i.e., $\mu_{i}=2 j \pi / N$, for all the positive integers for which $-\pi \leqslant \mu_{i} \leqslant 0$. Sengupta's method had been applied to determine the natural frequencies at which $\mu_{i}$ have the above values. In both the cases excellent agreement has been obtained in this case with the Arnold-Warburton's method (Figs 7-8). The values obtained by the wave propagation method are within less than $\pm 1 \%$ of the values obtained from Arnold-Warburton's method. The lowest bounding frequency is observed at $\mathrm{N}=18$ in both methods for Case 1 (Fig. 7). The lowest point for $\Omega_{N}$ against nodal patterns $N$ for Case 2 using both the methods (Fig. 8) is observed at $N=6$. Further investigation is required to determine which of the curves represent flexural and shear waves.

\section{Conclusions}

The excellent comparisons in the natural frequencies obtained by modified Mead and Bardell's method and Arnold-Warburton's method hereby prove the suitability of applying the wave propagation method to the study of free vibration of shell-type structures. It also proves that consideration (assumption (ii)) of the shell segment in between two consecutive circumferential nodes as a periodic structure is valid. The results from the wave propagation method are found to compare identically with the classical methods because both the methods lead to the exact solution of the same problem. Also a variation of the phase constants at the lower bounding frequency for the first propagation band for different nodal patterns has been observed. This method has enormous utility in analysis of periodic structures, in particular the wave propagation in shell structures and for the study of vibration characteristics. Hereby we can conclude that by considering a curved panel as a periodic shell element we can effectively analyze the complete shell structure. The significance of this elegant method lies in its simple final matrix forms that are easy to formulate and program. The method is highly computationally efficient. An attempt is presently underway to compare the results of the Mead and Bardell's approach for stiffened shells to Arnold-Warbuton's method. This study can be extended to study the shell flutter problem using the wave propagation approach [4]. Further investigations are currently underway to validate Mead and Bardell's results for stiffened shell with the classical method and extend it to study flutter.

\section{Acknowledgement}

The first author would like to thank Prof. S. Parthan of Dept. of Aerospace Engineering, IIT, Kharagpur, for his advisement on his MS Thesis.

\section{References}

[1] A.W. Leissa, Vibration of Shells, NASA SP-288, 1973.

[2] A.J. Dekker, Solid State Physics, Macmillan Press, London, 1981.

[3] A. Ghoshal, M.L. Accorsi and M.S. Bennett, Wave Propagation in Circular Cylindrical Shells with Periodic Axial Curvature, Wave Motion 23 (1996), 339-352.

[4] A. Ghoshal, Study of Free Vibration of Circular Cylindrical Shell using Wave Propagation, M.S.Thesis, Dept. Of Aerospace Engineering, IIT, Kharagpur, Dec, 1992.

[5] C. Avallet and J. Parot, Rechere d'ondes naturelles de coques cylindriques radies periodiquement, Revue du Cethedec 19 (1982), 159-173.

[6] C. Hodges, J. Power and J. Woodhouse, The low frequency vibration of a ribbed cylinder, Part 1: Theory, J. Sound and Vibration 101 (1985), 219-235.

[7] D.J. Mead and N.S. Bardell, Free Vibration of a Thin Cylindrical Shell with Discrete Axial Stiffeners, Journal of Sound and Vibration 111(2) (1986), 229-250.

[8] D.J. Mead, Free Wave Propagation in periodically supported, infinite beams, Journal of Sound and Vibration 11(3) (1970), 181-197.

[9] D.J. Mead, A general theory of harmonic wave propagation in linear periodic systems with multiple coupling, Journal of Sound and Vibration 27 (1973), 235-260.

[10] D. Li and H. Benaroya, Dynamics of periodic and near periodic structures, Appl. Mech. Rev. 45(11) (1992), 447-459.

[11] D. Photiadis, Wave mixing effects on a periodically ribbed cylindrical shell, ASME Winter Annual Meeting 1993, 93WA/NCA-23, pp. 1-10.

[12] G. Sengupta, Natural flexural waves and the normal modes of periodically-supported beams and plates, Journal of Sound and Vibration 13 (1970), 89-101.

[13] G. Warburton, Dynamics of Shells, Symposium of Structural Dynamics, Loughborough University of Technology, 1970.

[14] J.L. Sewall and Egle, An Analysis of Orthogonally Stiffened Cylindrical Shells with Stiffeners treated as Discrete Elements, AIAA Journal 6(3) (March 1968).

[15] K. Forsberg, Influence of Boundary Conditions on the Modal Characteristics of Thin Cylindrical Shells, AIAA Journal 2(12) (1964), 2150-2157.

[16] L. Brillioun, Wave Propagation in Periodic Structures, Electric Filters and Crystal Lattice, Dover Publications Inc, USA, 1953.

[17] M.L. Accorsi and M.S. Bennett, Wave Mode Conversion in Stiffened Cylindrical Shells with Periodic Axial Curvature, Journal of Vibration and Acoustics 119 (1997), 180-184.

[18] M.S. Bennett and M.L. Accorsi, Free Wave Propagation in Periodically Ring Stiffened Cylindrical Shells, Journal of Sound and Vibration 171 (1993), 49-66.

[19] N.S. Bardell and D.J. Mead, Free Vibration of an Orthogonally Stiffened Cylindrical Shell, Part-I: Discrete Line Simple Supports, Journal of Sound and Vibration 134(1) (1989), 29-54. 
[20] N.S. Bardell and D.J. Mead, Free Vibration of an Orthogonally Stiffened Cylindrical Shell, Part-II: Discrete General Stiffeners, Journal of Sound and Vibration 134(1) (1989), 55-72.

[21] N.S. Bardell and D.J. Mead, Free Vibration of a Thin Cylindrical Shell with Periodic Circumferential Stiffeners, Journal of Sound and Vibration 115(3) (1987), 499-520.

[22] R.N. Arnold and G.B. Warburton, Flexural Vibrations of Thin Cylindrical Shells Having Freely Supported Ends, Proc. Roy. Soc. London, Ser. A 197 (1949), 238-256.

[23] S. Markus, The Mechanics of Vibration of Cylindrical Shells, Elsevier, New York, 1988.

[24] S. Parthan and D.J. Johns, Vibration and Flutter of Unstiffened and Orthogonally Stiffened Circular Cylindrical Shells, (Vol.I-II), Department of Transport Technology, Loughborough University of Technology TT7106, June 1971.

[25] W. Flugge, Stresses in Shells, Springer Verlag, Berlin, 1960.

\section{Appendix A: Periodic system and Bloch (Floquet's) Theorem}

A periodic system consists of a number of identical elements, joined together in an identical manner (endto-end or side-to-side) to form the complete system. The periodicity may depend on geometrical and material similarity [10]. The electronic motion in a constant and a periodic potential is analogous to the propagation of elastic waves in a continuum and in a periodic structure. For elastic waves in a continuous medium, the frequency is inversely proportional to the wavelength i.e., there exists a linear relationship between frequency and wave number or wave vector. This implies that the velocity of propagation is independent of wavelength. Furthermore theoretically, there exists no upper limit for the frequency of the vibrational modes in a continuous medium. However, when one considers the modes of vibration in a lattice of discrete mass points, which form a periodic structure, two characteristic features appear: (a) There exists allowed frequency (propagation) bands separated by forbidden regions (attenuation). (b) The frequency is no longer proportional to the wave number but a periodic function of the latter.

In a constant potential (free electron theory), the energy of the electron as function of the wave vector $k$, is given by $E=\frac{h^{2} k^{2}}{2 m}$, where $k=2 \pi / \lambda=p / h$. Here $\lambda$ is defined as the wavelength associated with the electron and $p$ is the momentum of the electron. Potential energy is assumed to be zero. Considering the motion of an electron in a periodic potential, one can infer that: (a) there exist allowed energy bands (propagation) separated by forbidden regions (attenuation), and (b) the functions $E(\bar{k})$ are periodic in $\bar{k}$. One can derive the Schrodinger equation for an electron moving in a one-dimensional periodic potential and by letting the potential energy satisfy the equation, as:

$$
d^{2} \Psi / d x^{2}+\left(2 m / h^{2}\right)[E-V(x)] \Psi=0
$$

Here $E$ is the kinetic energy of the electron and $V(x)$ is the periodic potential energy i.e, $V(x)=V(x+a)$ where $\mathrm{a}$ is the period. Bloch Theorem states that there exist solutions for Eq. (1) of the form

$$
\begin{aligned}
& \Psi=e^{ \pm i k x} u_{k}(x) \quad \text { where } \\
& u_{k}(x)=u_{k}(x+a)
\end{aligned}
$$

The solutions are plane waves modulated by the function $u k(x)$ which has the same periodicity as the lattice. In the theory of differential equations, Bloch Theorem is known as Floquet's Theorem. Functions of the type of Eq. (2) are known as Bloch's functions. Details of the derivations and discussions of Bloch's functions and Schrodinger's equation for the energy spectrum of an electron in a periodic potential consisting of allowed and forbidden energy or pass and stop bands can be found in Refs [2,16].

Energy carrying flexural waves in periodic beamtype structures have received considerable attention from researchers in the past. If the infinite periodic beam is undamped, free harmonic wave motion without decaying is possible in the absence of forcing from an external pressure field (i.e., no external forces acting on an element apart from those coming from the two adjacent elements). It had been long known that waves can only propagate freely in certain distinct frequency bands [8]. At frequencies outside these propagation bands, a wave, once started, will decay as it spreads outwards. In fact, there exist alternate bands of free propagation and decay. The upper and lower limits of these frequency bands are particularly significant. Infinite periodic beams and plates possess the further property that the bounding frequencies are identical with the natural frequencies of a single element, with its ends either simply supported or fully fixed. The propagation constants have been defined as the characteristics of wave motion and are measures of the rate of decay and change of phase of the wave motion over the distance between adjacent elements.

The free wave motion is not of a simple spatially sinusoidal form, as the supports cause reflections. Nevertheless, free waves of distinctive type can be recognized, and wave velocities identified for given frequencies. A beam on rigid supports has a single propagation constant for each frequency, and a unique wave group with sinusoidal wave components of different wavelengths, phase velocity, and direction. Wave motions in 
periodic plates are, in general, much more complicated than in beams. Waves over flat surfaces can spread out in a manner analogous to circular waves, or in a simple form analogous to plane waves. Waves of the first type could be generated by a single point source (say, an oscillating force) acting on one of the periodic elements and propagating energy out into all directions. The wave intensity may be greater in some directions than in others, depending on the geometry and structural characteristics. Waves of the second type, which are analogous to plane waves, have been described by Mead [9]. They could be generated by a certain distribution of force along a straight line at any angle across the periodic structure. All the periodic elements then vibrate in identical complex harmonic modes, but a phase difference exists between the motions of corresponding points in adjacent elements, which are the same for any pair of elements. A detailed study of dynamics of periodic and near-periodic structures had been done in Ref [10]. 

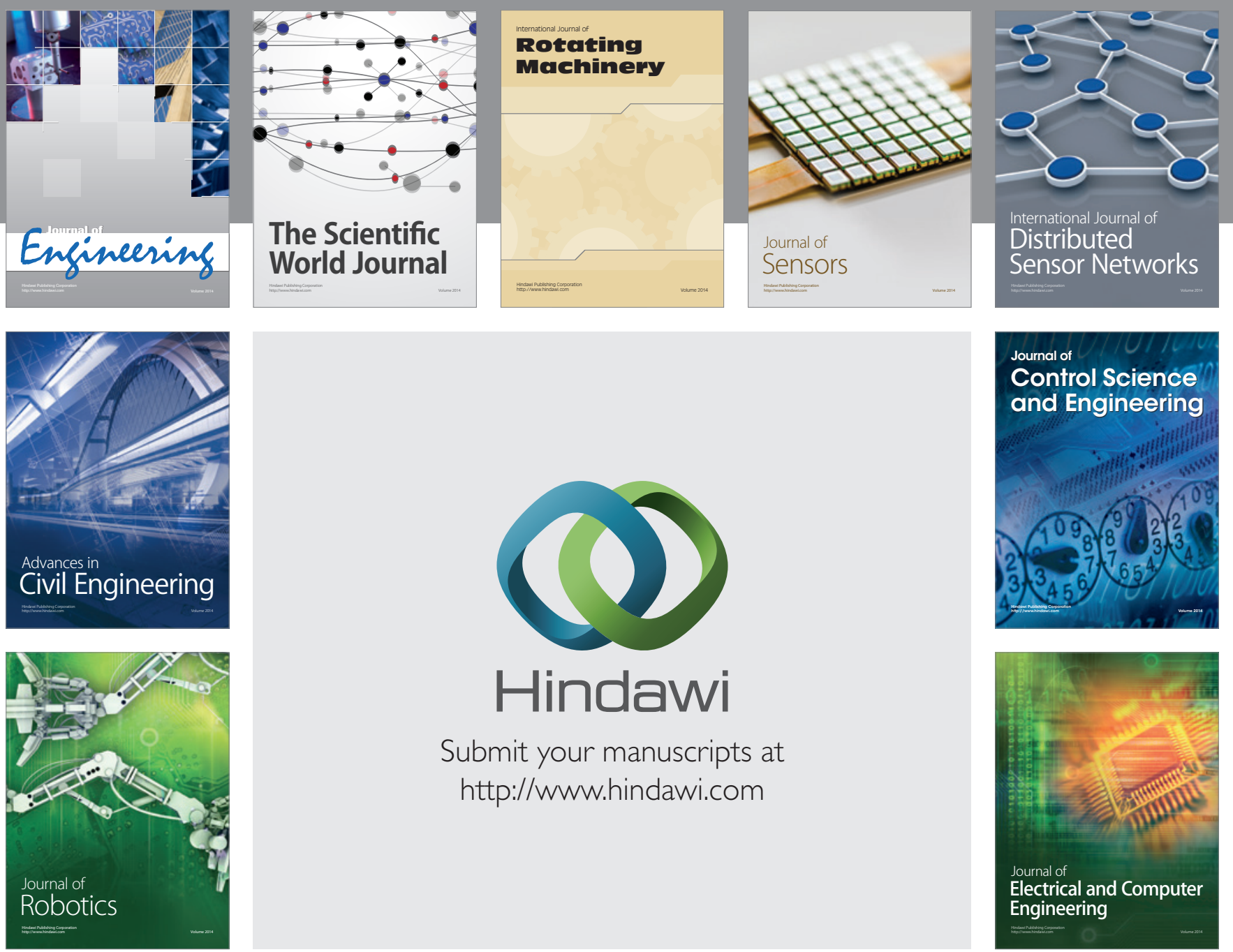

Submit your manuscripts at

http://www.hindawi.com
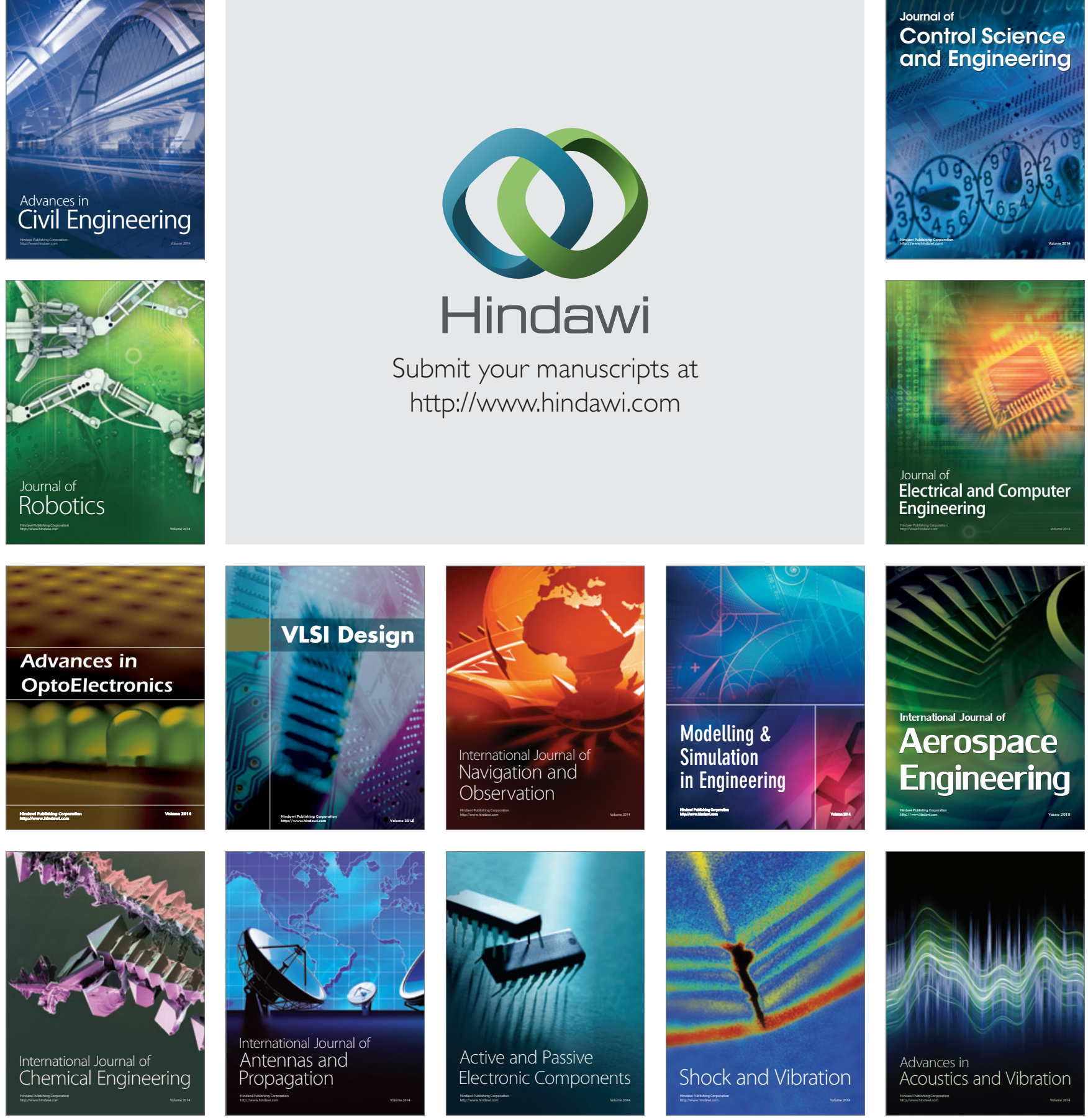